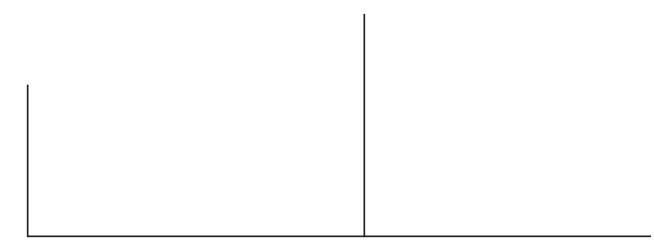

\title{
Avatares en la clínica del sujeto y el destino: una experiencia mexicana
}

\author{
Francisco Landa Reyes
}

\begin{abstract}
Se presentan algunas reflexiones en torno a la constitución en México de una Asociación Civil dedicada a la atención de pacientes con padecimientos psíquicos graves. Los aspectos tratados son: La propuesta de una intervención interdisciplinaria que centre su atención en el trabajo de caso y en la responsabilidad ótica por el tratamiento, la necesidad de una teorización sobre el destino del sujeto en la psicosis, y la conceptualización del acompañamiento terapéutico como intervención clínica.
\end{abstract}




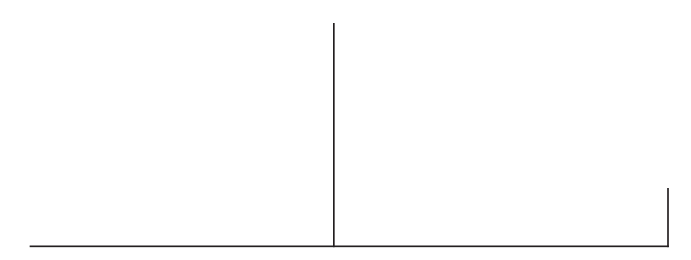

\section{El contexto de la atención a la psicosis en México}

Quizá el panorama de la atención a la psicosis en México no sea tan difícil de resumir en unas cuantas líneas. Primeramente habría que decir que en cuanto a la atención ofrecida por las instituciones del Estado, la situación es prácticamente la misma desde hace algunas décadas: los hospitales, saturados, a penas logran ofrecer una contención física y farmacológica a los pacientes, que ven reducidas sus perspectivas a una permanencia perenne o intermitente en los programas asistenciales. Es apenas en los últimos dos años que se han comenzado a implementar cambios. Los hospitales psiquiátricos del Estado intentan revertir su deterioro bajo la óptica de una optimización de la calidad de sus servicios; sin embargo, tales reformas aún no hallan una aplicación total. Además, no las respalda un modelo clínico de rehabilitación a partir del cual se pudiera pensar en un revertimiento efectivo de la cronificación de los pacientes hacia posibilidades reales de integración familiar, comunitaria y laboral.

Sin dejar de considerar la complejidad que implica el trabajo con pacientes graves, resulta evidente que en las instituciones estatales - pero también privadas -, no se cuenta con estrategias de intervención que le permitan a los pacientes:

a) Lograr una estabilización duradera con ausencia de cuadros agudos.

b) Consolidar una inserción efectiva en su entomo social. 


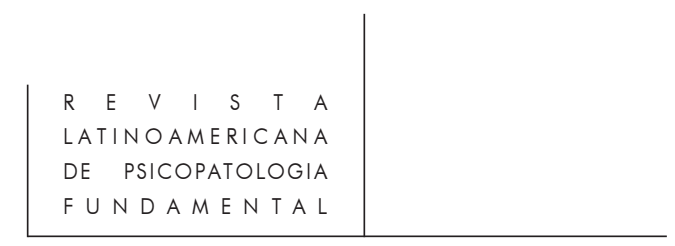

c) Contar con la perspectiva de un porvenir que no esté determinado absolutamente por un diagnóstico inhabilitador.

La estrategia vigente de los hospitales, cuyo criterio para la alta de los pacientes es la remisión de los síntomas, deja a un lado que tal indicador no implica que el sufrimiento haya terminado, ni que la problemática a la que se tengan que enfrentar al salir no siga allí.

Tenemos así un impasse: Aún cuando el tratamiento médico hospitalario sea aplicado adecuadamente, existe una carencia de atención a los aspectos psíquicos y sociales de los padecimientos, carencia que ni los médicos ni las instituciones pueden cubrir actualmente, pues excede sus posibilidades, sobre todo considerando la situación crítica de las partidas presupuestarias gubernamentales.

A este contexto, quizá demasiado pesimistamente planteado pero que no deja de ser realista, hay que añadir durante los últimos años la participación cada vez más activa de la sociedad civil en México.

Desde los sismos de 1985, el movimiento democrático de 1988, y hasta la conformación del zapatismo civil y de un congreso plural en 1994, la sociedad mexicana ha generado una serie de transformaciones cuyo sentido es una participación cada vez más activa en la vida del país, y una de cuyas tendencias 124 es la fiscalización de las tareas e instituciones del gobierno, pero en el límite, incluso el relevo de sus funciones por grupos civiles organizados que toman a su cargo lo que la institución gubernamental es incapaz de solventar.

Dentro del ámbito de la atención psiquiátrica, ambas formas de intervención de la sociedad civil han operado en México: instituciones como el Capítulo Mexicano de la Asociación Mundial de Rehabilitación Psicosocial han presionado y conjuntado esfuerzos para aumentar la fiscalización y colaboración de grupos civiles en torno a hospitales psiquiátricos en pro de una estricta observancia a los derechos humanos y de una prevalencia del enfoque rehabilitatorio por encima del asilar.

Pero también ha habido por parte de otras agrupaciones civiles esfuerzos tendientes a ofrecer por ellas mismas atención a los pacientes con episodios de psicosis. Tal es el caso de Centro Montealbán, agrupación de la que forma parte el que suscribe. Este grupo comenzó como una red de profesionales con experiencia en instituciones estatales, privadas y en la atención de gabinete, y se fue transfomando en una opción que ofrece un tratamiento integral alterno para los pacientes tradicionalmente derivados a los hospitales psiquiátricos. 


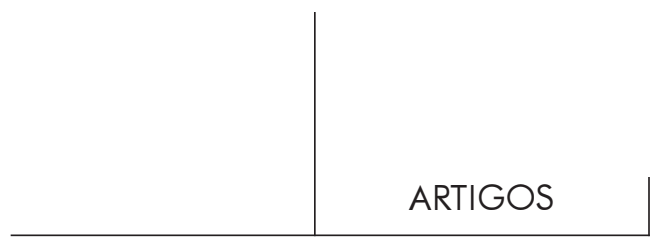

\section{La experiencia del Centro Montealbán}

Quizá la recuperación de la experiencia de Centro Montealbán resulte valiosa para situaciones similares en otras geografías, por ello incluimos aquí algunos detalles sobre su conformación.

A partir del trabajo en pequeños equipos conformados alrededor del tratamiento de algunos pacientes, en algunos casos junto con sus familias, se gestaron también seminarios públicos en los que se reflexionaba sobre el tratamiento posible de la psicosis y el establecimiento de un modelo de atención y cuidado a los pacientes. El grupo fue creciendo, así como el número de pacientes atendidos.

Posteriormente se conformó legalmente una Asociación Civil sin fines de lucro dedicada a ofrecer tratamiento médico, psicoterapéutico y rehabilitatorio a pacientes con padecimientos psíquicos graves (principalmente psicosis).

Lo que ha caracterizado el funcionamiento de este grupo ha sido la pluralidad. Así, desde cada disciplina se ha ido perfilando un estilo de trabajo.

Desde una mirada médica, psiquiatras con una amplia experiencia de trabajo dentro y fuera de los hospitales, apuestan por una forma de intervenir en la que el tratamiento farmacológico sea parte fundamental pero no definitoria de la atención a los pacientes. Este grupo de médicos parte de la necesidad de un trabajo en conjunto con psicoanalistas y otros psicoterapeutas, así como de contar con el apoyo de otros agentes que faciliten la integración familiar y social del paciente. En el campo de la farmacologia, se comprometen con un uso responsable de los medicamentos, de modo que éstos sean propiciadores de la estabilización psíquica sin llegar a convertirse en bloqueadores de la aparición de la palabra de los pacientes.

Por otra parte, con una fuerte influencia de las formulaciones psicoanalíticas de S. Freud y J. Lacan, pero sin que este enfoque teórico les sea exclusivo, un amplio grupo de psicólogos y psicoanalistas realizan un intenso trabajo clínico con vistas a ofrecer a los pacientes un espacio para la elaboración de sus interrogantes vitales. Dar la palabra al paciente para que hable de su sufrimiento y de su experiencia singular, para que elabore la pregunta por la causa de su padecimiento, para que pueda historizar sus experiencias subjetivas y darles un sentido, son los ejes de esta psicoterapéutica basada en la escucha. A partir de tal intervención, se pretende que el paciente esté en posición de situar su pasado, transformar el presente y proyectar su futuro, convirtiéndose en agente deterinador de su destino.

Por último, un amplio conjunto de actores sociales que incluye a psicólogos, artistas, docentes y otros, se encuentra trabajando en la creación de espacios que favorezcan la integración de las personas con padecimientos psíquicos graves 


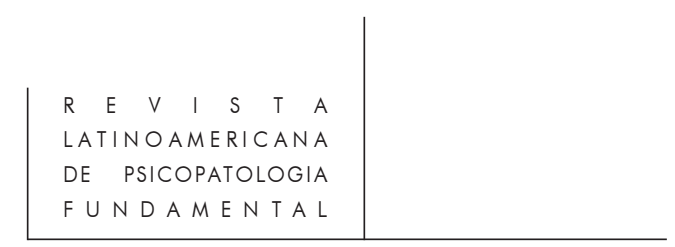

a actividades formativas, productivas y creativas. Desde una multiplicidad de talleres artísticos y laborales, hasta el establecimiento de espacios integrados a centros educativos y laborales, se hacen intentos por contrarrestar la severa desconexión de todo lazo social que se da como consecuencia de múltiples cuadros patológicos. Este tipo de esfuerzos, además de brindar apoyo a los pacientes para reanudar sus relaciones familiares, afectivas y laborales, están favoreciendo la creación de una nueva cultura de la integración al interior de nuestras comunidades. De la conjunción de estas tres vertientes ha surgido Centro Montealbán.

Pero hay que añadir otro dato importante: esta agrupación ha sido también el espacio donde se ha consolidado como una práctica efectiva en el tratamiento de la psicosis y otros padecimientos graves un perfil novedoso: el Acompañamiento Terapéutico.

\section{El Acompañamiento Terapéutico: una nueva práctica}

Surgido inicialmente como una práctica difusa que se adaptaba a diversas necesidades de apoyo extra-consultorio, al trabajo de psicoterapeutas, y al sostenimiento de las actividades de las primeras comunidades terapéuticas surgidas en los años setenta, el Acompañamiento Terapéutico ha venido cobrando especificidad como una práctica clínica cada vez más diferenciada.

Si bien su conceptualización es incipiente, y su fundamentación puede partir de marcos teóricos diversos - o incluso prescindir de ellos -, como práctica efectiva llevada a cabo por profesionales y semiprofesionales se encuentra en franca expansión tanto en México como en otros países. Su principal ventaja es que extiende los efectos curativos de fármacos y psicoterapias espacial y temporalmente, y representa una estructura intermedia de atención que amortigua la salida del paciente del hospital y previene su crónico reingreso al mismo.

En términos prácticos, el Acompañamiento Terapéutico consiste en la cercania consuetudinaria de uno o varios acompañantes a uno o varios pacientes durante sus actividades cotidianas. Ya sea que el paciente se encuentre en su domicilio o en lugares públicos, e incluso cuando es posible y necesario en su escuela, trabajo o sitio de hospitalización, el acompañante está con él realizando actividades en conjunto, dialogando, compartiendo.

A partir de una fundamentación psicoanalítica del Acompañamiento, que es la que a juicio del autor permitiría diferenciarlo claramente de otras prácticas similares como la de los enfermeros y garde-fous, es posible establecer una serie de coordenadas definitorias de esta intervención clínica.

En primer lugar, el Acompañamiento Terapéutico, dentro de un marco analítico, se define por ser una intervención necesariamente incluida en un 
dispositivo interdisciplinario de atención que incluya además al Psiquiatra y/o Psicoterapeuta a cargo del tratamiento. Por formar parte del equipo tratante, el acompañante se torna corresponsable no solo de la integridad física o del estado emocional del paciente, sino de su tratamiento en un sentido amplio. Del mismo modo, su inclusión al equipo tratante le permite conocer aspectos fundamentales de los factores genéticos, históricos y relacionales que determinan el estado actual del paciente, así como sus perspectivas de estabilización y reinserción social y familiar.

A partir del sentido que puede adquirir su participación en el equipo tratante, y de la relación que establezca con el propio paciente, el acompañante tiene como tareas centrales en su hacer cotidiano:

a) La contención del paciente a diversos niveles: Simbólico con su palabra, Imaginario con su mirada, Real con sus actos. Contener significa evitar el desbordamiento que en la alucinación, el delirio o el pasaje al acto puede llevar al paciente a su autodestrucción.

b) El reanudamiento de los lazos sociales del paciente. El acompañante tiene como función una cierta intermediación no suplementaria de la palabra del paciente que le permita a éste recuperar u obtener un lugar en su familia y en su comunidad, superando los efectos de exclusión y marginación que forman parte de la lógica de su padecimiento.

c) La construcción de espacios en la realidad intersubjetiva para ser habitados por el paciente. El acompañante, junto con él y a partir de la escucha de su dimensión deseante y de la ponderación de sus posibilidades reales, y en su calidad de participante de una red comunitaria, propicia creativamente su integración social.

La directriz ética que da sentido a éstas dimensiones fundamentales del Acompañamiento es la finalidad de brindarle al paciente un lugar de sujeto con una palabra, un conjunto de derechos ciudadanos, así como la posibilidad de hacerse responsable de sus actos.

\section{Retos y perspectivas de Centro Montealbán}

Como se puede observar, la perspectiva de Centro Montealbán ante su constitución como Asociación Civil que ha tomado a su cargo la atención de pacientes con padecimientos psíquicos graves es muy compleja. Quizá el asunto de mayor dificultad sea la diversidad de disciplinas y personas confluyendo con un mismo objetivo.

El campo de discusión que se crea abarca muchas formas de abordaje teórico-metodológico. Además, a las dificultades dadas por la constitución 


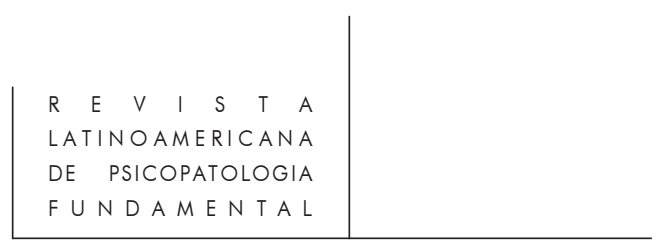

interdisciplinaria del equipo, hay que añadir aquellas determinadas por el contexto sociopolítico y por las necesidades prácticas que impone la conversión de una red informal en una institución.

Existen tres necesidades fundamentales en este momento para Centro Montealbán:

1. Organizar y en su caso obtener los recursos humanos, materiales y monetarios necesarios para su funcionamiento.

2. Sostener un diálogo interdisciplinario e interinstitucional que permita la cooperación intra y extragrupal; diálogo que debe evitar la confrontación ideológica y la pugna territorial por cotos de poder.

3. Desarrollar las elaboraciones teóricas que sustenten y permitan depurar el modelo de intervención, especialmente la poco conceptualizada práctica del Acompañamiento Terapéutico.

Hasta el momento, la resolución de estas necesidades se encuentra así:

Por lo que toca a las exigencias que impone la institucionalización, ha sido necesario elaborar una estrategia de financiamiento que resuelva el sostenimiento de un espacio que funcione como Centro de Atención que unifique las actividades que de manera dispersa realizan hasta hoy los miembros de la institución:

128 Psiquiatras, Terapeutas, Acompañantes y Talleristas. En el contexto económico de nuestro país y dada la intención de mantener un servicio accessible a todos los estratos sociales, tal tarea no es nada fácil. Sin embargo su resolución marcha en tanto se puede hacer mucho con poco, y los espacios disponibles para el uso entre los que se cuentan los consultorios de los integrantes así como instalaciones públicas bastan por el momento para los fines de Centro Montealbán, excluyéndose la recepción 24 horas y la posibilidad de brindar alojamiento.

Por otra parte, en relación al diálogo interdisciplinario y las dificultades que impone, el camino tomado hasta hoy ha requerido superar las diferencias que en el nivel teórico-metodológico han fragmentado tradicionalmente la atención a pacientes psicóticos en parcelas disciplinarias.

Ante ésto, la propuesta ha sido, en tanto su compleja realidad lo permita, abordar la psicosis no como un objeto de estudio del cual la posesión es disputada, sino como un enigma cuya inaccesibilidad atañe a los límites del conocimiento para explicar la cosa misma, y del cual no cabe un desciframiento, sino una discreta búsqueda de los modos como podemos ponernos en relación con él. En tanto que la composición de los grupos de trabajo lo permita, la idea ha sido acercarse a la psicosis en conjunto, intentando hacer converger diferentes discursos y aproximaciones.

La diversidad de las concepciones sobre la psicosis - aún al interior de cada disciplina - coloca en un campo de discusión muy amplio que abarca temas 
teóricos, pero también políticos y éticos. Entonces se propone que lo que debe guiar la discusión es la clínica misma: el trabajo de casos, la discusión de tácticas y estrategias. Un tal modelo de intervención suponde, entre otras cosas, asumir la incompletud de toda construcción teórica para dar cuenta de la totalidad del campo de la psicosis, así como la puesta a un mismo nivel de los discursos tradicionales sobre el tema. Del mismo modo, se asume la imposibilidad de totalizar el campo por el artilugio de la via ecléctica, pues no es posible suponer a la verdad como resultado de la suma de los saberes.

Se supone y se practica hasta donde es posible el acudir a teorías etiológicas y nosológicas sólo para después poder adentrarse en la discusión clínica singularizada basada tanto en la mirada médica como en la escucha analítica de cada sujeto. Pero ello requiere, y éste es el paso más delicado, clarificar y hacerse cargo de la discusión ética que partiría de la explicitación de las ideas de la cura o tratamiento posible de la psicosis que se tengan.

La confluencia de teorías y metodologías solamente es planteable con sensatez si se parte de la posibilidad de una convergencia ética. En Centro Montealbán nos parece requisito indispensable partir del supuesto de que con la psicosis se puede hacer algo, que no es un fenómeno del reino de lo necesario, de lo absolutamente determinado. Y es que aún en el caso de las psicosis orgánicamente determinadas, la pregunta sigue siendo, como diría M. Silvestre: ¿Y qué puede hacer el sujeto ante lo que lo determina?

Esto nos lleva a una última reflexión sobre nuestro requerimento de elaborar el background teórico que sustente nuestro modelo de atención.

Efectivamente, si la posibilidad de superar las diferencias teóricometodológicas en aras de un trabajo interdisciplinario parte del trabajo detallado de caso en el que se expliciten las posiciones éticas, es decir las concepciones sobre los alcances y la finalidad del tratamiento, entonces estamos ante la necesidad de contar con una serie de referentes, si no es que con una teoría sobre el sujeto y el destino en la psicosis.

Quizá no haya práctica clínica dentro del campo de la psicopatología en la que estemos más comprometidos con nuestra palabra que en la atención a la psicosis. La transmisión al paciente, explícita o implícitamente, de un pronóstico para su padecimiento, puede convertirse en palabra fatal que coloque una loza sobre toda esperanza de vida de un sujeto con una voz, un deseo y un futuro. Un diagnóstico que conduzca de manera automática a un pronóstico inhabilitador del tipo: "usted no podrá casarse, enseñar, estar sin medicamentos ..." muestra antes que nada una posición de quien la enuncia ante el destino humano, pero además un desprecio y un desconocimiento de la apertura del campo de lo posible en la singularidad de cada caso. 


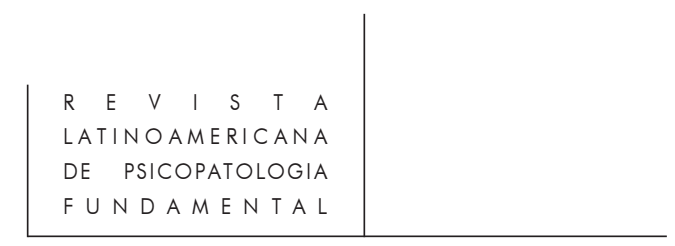

De nuestra concepción del destino del sujeto de la psicosis dependerá el posible tratamiento de cada uno de nuestros pacientes. Por ello, se hace necesario contar con una teoría del sujeto en la psicosis que permita sostener teóricamente, más allá de las pugnas ideológicas, la factibilidad de una emergencia de sujeto en el sentido antiguo de la palabra: el hypokeimenon griego (quien está detrás de la decision y del acto). Si partimos de la posibilidad - si bien en una baja probabilidad - de que quien padece episodios psicóticos pueda responder de sí, entonces una clínica de la psicosis y un modelo como el que proponemos podrá sostenerse.

\section{Conclusión}

Para finalizar cabe decir que quizá lo que pueda aportar la experiencia Montealbán desde el contexto mexicano sea el intento, con todo y sus avatares, de lograr una atención a los pacientes con padecimientos psíquicos graves que les permita a éstos, si lo desean, tomar el lugar de sujetos responsables y agentes de su destino.

Tal intento, por tomar la dimensión de una propuesta de la sociedad civil para la sociedad civil, y por constituirse como alterno a la atención de las instituciones del Estado, cobra una mayor especificidad en las dificultades que enfrenta.

Pero quizá lo más interesante sea cómo este proyecto apuesta tercamente por el trabajo interdisciplinario, y paga por ello el costo de tener que abandonar la comodidad de las posiciones teóricas establecidas para, sin caer en el eclecticismo, proponer una fundamentación responsable de su modelo de atención.

Del trabajo fino caso por caso desde las diferentes aproximaciones metodológicas hasta la construcción junta con el paciente de un destino posible construyéndose cada día acompañándolo en su devenir, quizá no sea tan descabellado descartar toda preconcepción de lo que va a suceder.

Quizá baste con iniciar por hacerse preguntas como: ¿Qué tipo de vida creemos que puede construir nuestro paciente? ¿Qué grado de remisión de síntomas es el adecuado que provea el medicamento? ¿Qué espacio dejar al delirio? ¿Cómo pensar a este en relación con el contexto social del paciente? ¿De qué manera trabajar con la familia? ¿En qué momento es necesaria una internación hospitalaria? ¿Qué ámbitos de escucha corresponden a cada uno de los intervinientes en el tratamiento, a partir de su estilo, posibilidades y relación con el paciente? ¿Cómo trabajar con la familia y el paciente mismo la pregunta por el origen de su padecimiento?

El compromiso del equipo tratante es responder a estas preguntas con claridad, y procesar las diferencias que se presenten en su resolución. En esto 
consiste la dirección del tratamiento, más allá de quién sepa más o menos, una teoría u otra. La dirección no puede ni debe consistir en un saber anticipatorio, sino en una responsabilidad a la vez del equipo y de cada uno de sus miembros sobre las acciones terapéuticas.

Trabajar en equipo con una fundamentación ética, significaría poder hacerse responsables ante y con cada paciente de la dirección de su tratamiento. Significa reconocer en la psicosis un campo en el que las limitaciones son mucho mayores que las esperanzas. Pero también, y sobre todo, significa estar abiertos a que el paciente y su familia encuentren nuevos caminos, a acompañarles en su búsqueda al menos no hacióndose cómplices de la cosificación y el borramiento subjetivos.

La idea que ponemos a prueba y a consideración, y cuyos resultados hasta ahora nos mantienen en la brecha, es que la intervención del equipo interdisciplinario puede permitir a cada paciente que habita la psicosis abrir una vía para su permanencia en el mundo, abrir un espacio para la escritura de su destinar.

Se trata, quizás, de convertir el estrecho resquicio de lo posible que nos deja la psicosis en un campo de invención responsable. De destinar, en todo caso, junto con nuestros pacientes. Destinar como fin, destinando como medio. Pues la historia del tratamiento de la psicosis también se está escribiendo. Escribirla.

\section{Bibliografia}

Del Collado, F. (1997). "Hospitales psiquiátricos: la razón ante la locura". Suplemento enfoque del diario Reforma, 2/Noviembre/1997, 9-10.

LANDA, F. (1997). "Quelques idées generales pour définir l'accompagnement thérapeutique à partir de la psychanalyse". Texto presentado en el Primer encuentro de intercambio Sainte-Anne-Centro Montealbán. Universidad Autónoma Metropolitana, México.

EQUIPO INTERDISCIPLINARIO DE ATENCIÓN A LA PSICOSIS (1998). "Elementos para una articulación de las cuestiones éticas y clínicas". Trabajo presentado en el Primer Coloquio sobre Modelos de atención a la Psicosis organizado por Centro Montealbán. Universidad Autónoma Metropolitana, México.

Morales, H. (1997). Sujeto y estructura. México, Ediciones de la Noche.

Rossi, G. y Pulice, G. (Comp.) (1990). Primer congreso sobre acompañamiento terapéutico. Buenos Aires, Javier Bóveda.

Strauss, J. (1997). "La nature de la schizophrénie: vulnerabilité et destin". L'évolution psychiatrique, 62, no 2, 245-262. 


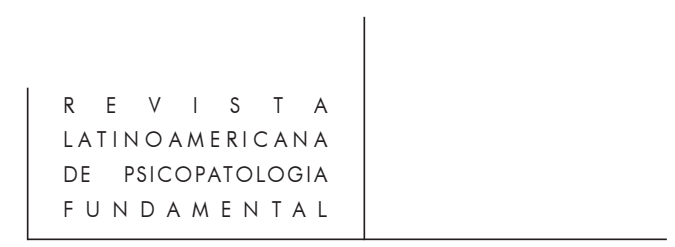

\section{Resumos}

São apresentadas algumas reflexões sobre a constituição, no México, de uma Associação Civil dedicada a pacientes com doenças psíquicas graves. Os aspectos tratados são: a proposta de uma intervenção interdisciplinar que centre sua atenção no estudo de caso e na responsabilidade ética pelo tratamento; a necessidade de uma teorização sobre o destino do sujeito na psicose e a conceitualização do acompanhamento terapêutico como intervenção clínica.

Cet article présente quelques élaborations au tour de la création au Mexique d'une Association Civile dediée au soin des patients aux troubles psychiques graves. Les aspects traités sont les suivants: la proposition d'une intervention pluridisciplinaire dont le noyau serait le travail de cas, et dont la direction serait donnée par la responsabilité éthique du traitement; la théorization à propòs du destin du sujet dans la psychose, et l'accompagnement thérapeutique comme intervention clinique.

Some reflections upon the constitution, in Mexico, of a Civil Association dedicated to patients with severe psychic disturbances are here presented. The aspects dealt with are: the proposal of an interdisciplinary intervention that focuses its attention on cases studies and on the ethical responsibility for treatment; the necessity of a theorisation on the fate of the subject in psychosis; and the conceptualisation of therapeutic follow-up as a clinical intervention. 